OPEN ACCESS

Edited by:

Thomas A. Ficht,

Texas A \& M University, United States

Reviewed by:

Maria Isabel Colombo, Universidad Nacional de Cuyo,

Argentina

Erdong Cheng, University of Pittsburgh Cancer

Institute, United States

*Correspondence:

Ge Zhang

zhangge@mail.sysu.edu.cn

tThese authors have contributed equally to this work

Received: 04 December 2017 Accepted: 29 May 2018

Published: 19 June 2018

Citation:

Feng X, Lu J, He Z, Wang Y, Qi F, Pi R and Zhang $G$ (2018) Mycobacterium smegmatis Induces Neurite Outgrowth and Differentiation in an Autophagy-Independent Manner in PC12 and C17.2 Cells. Front. Cell. Infect. Microbiol. 8:201. doi: 10.3389/fcimb.2018.00201

\section{Mycobacterium smegmatis Induces Neurite Outgrowth and Differentiation in an Autophagy-Independent Manner in PC12 and C17.2 Cells}

\author{
Xinwei Feng ${ }^{1 \dagger}$, Junfeng $\mathrm{Lu}^{2+}$, Zitian $\mathrm{He}^{3}$, Yidan Wang ${ }^{4}$, Fangfang $\mathrm{Qi}^{3}$, Rongbiao $\mathrm{Pi}^{2}$ and \\ Ge Zhang ${ }^{1 *}$ \\ 'Department of Microbial and Biochemical Pharmacy, School of Pharmaceutical Sciences, Sun Yat-sen University, \\ Guangzhou, China, ${ }^{2}$ Department of Pharmacology and Toxicology, School of Pharmaceutical Sciences, Sun Yat-sen \\ University, Guangzhou, China, ${ }^{3}$ Department of Anatomy and Neurobiology, Zhongshan School of Medicine, Sun Yat-sen \\ University, Guangzhou, China, ${ }^{4}$ Department of Biotechnology, School of McCormick Engineering, Northwestern University, \\ Evanston, IL, United States
}

Both pathogenic and non-pathogenic Mycobacteria can induce the differentiation of immune cells into dendritic cells (DC) or DC-like cells. In addition, pathogenic Mycobacteria is found to stimulate cell differentiation in the nerves system. Whether non-pathogenic Mycobacteria interacts with nerve cells remains unknown. In this study, we found that co-incubation with fast-growing Mycobacteria smegmatis induced neuron-like morphological changes of $\mathrm{PC} 12$ and $\mathrm{C} 17.2$ cells. Moreover, the $M$. smegmatis culture supernatant which was ultrafiltrated through a membrane with a 10 kDa cut-off, induced neurite outgrowth and differentiation in an autophagy-independent pathway in PC12 and $\mathrm{C} 17.2$ cells. Further analysis showed that IFN- $\gamma$ production and activation of the PI3K-Akt signaling pathway were involved in the neural differentiation. In conclusion, our finding demonstrated that non-pathogenic $M$. smegmatis was able to promote neuronal differentiation by its extracellular proteins, which might provide a novel therapeutic strategy for the treatment of neurodegenerative disorders.

Keywords: Mycobacterium smegmatis, neurite outgrowth, differentiation, autophagy, PI3K-Akt pathway

\section{INTRODUCTION}

The microbiome has tremendous potential to influence human physiology (Sharon et al., 2014). Host-microbe interactions affect immunity, metabolism, development, and behavior (Vuong et al., 2017). Some bacteria can produce neuroactive metabolites, ranging from serotonin and $\gamma$-amino butyric acid, to dopamine and norepinephrine, to acetylcholine and histamine to influence the nervous system (Takahashi et al., 2012; Oleskin et al., 2017).

Recently, emerging researches have shown that dysbiosis of gut bacteria, commensal skin or other microbiota can affect host behavior by producing chemical signals or directly influencing host nervous systems (Ochoa-Reparaz and Kasper, 2014; Obata and Pachnis, 2016). Moreover, Microbiota have been identified within immune-privileged sites such as the central nervous system (CNS). Proteobacteria and Actinobacteria are reported to be the major commensals persistent in 
the human brain regardless of immune status (Branton et al., 2013).

Mycobacterium, a genus of Actinobacteria, is a highly diverse and comprises various different pathogenic and nonpathogenic species. Interestingly, although undergoing apoptotic death and necrosis, cell proliferation or differentiation was also found during the pathogenic Mycobacterium infection in nerves system (Rambukkana, 2010; Spanos et al., 2015). A previous study demonstrated that $M$. leprae reprogrammed Schwann cells, a type of glial cells in the peripheral nervous system, to a stage of progenitor/stem-like cells during the infection, and identified that $M$. leprae-infected Schwann cells increase cell proliferation by maintaining the infected cells in a de-differentiated state (Masaki et al., 2013). In addition, during $M$. tuberculosis infection, bacilli can be replicated within microglia, which appears to be damaged in the periphery, and could be profitable by modulating neuronal regeneration and differentiation (Spanos et al., 2015).

Additionally, environmental exposure to non-tuberculous mycobacteria is common. M. vaccae, a soil bacteria, can induce neurogenesis by stimulating the production of serotonin and norepinephrine in the brain, resulting in an antidepressant effect (Reber et al., 2016). Moreover, a polyketide toxin from $M$. ulcerans induced hypoesthesia, which leads to potassium-dependent hyperpolarization of neurons and blocking of neuronal signaling transduction, consequently annulling the pain of the lesions bacteria (Marion et al., 2014).

Mycobacteria smegmatis is a rapidly growing environmental species typically living in water and food sources, occasionally involving in skin or soft tissue infections. M. smegmatis was reported to induce the differentiation of human monocytes into mature dendritic cells directly (Martino et al., 2005). Surprisingly, we noticed that the infection of M. smegmatis induced neuronal morphology changes of PC12 cells. To elucidate the interactions between Mycobacterium and nerve cells, we investigated the morphological changes during infection and explored the mechanism of M. smegmatis-induced neurite outgrowth as well as neuronal differentiation.

\section{MATERIALS AND METHODS Cell Culture}

Rat adrenal pheochromocytoma cell line PC12 (Cell Bank of Chinese Academy of Sciences, Shanghai, China) were grown in RPMI 1640 (Invitrogen, USA) supplemented with 10\% horse serum (HS) and 5\% fetal bovine serum (FBS) (Gibco, USA). Murine neural stem cell line C17.2 (Mouse multipotent neural progenitor cells) were grown in DMEM (Invitrogen, USA) supplemented with $10 \%$ FBS. All of the cell lines were cultured in standard humidified incubators $\left(37^{\circ} \mathrm{C}\right.$ and $\left.5 \% \mathrm{CO}_{2}\right)$.

\section{Bacterial Culture}

M. smegmatis strain ATCC 700084 was purchased from the China General Microbiological Culture Collection Center (CGMCC, Beijing, China). M. smegmatis were grown at $37^{\circ} \mathrm{C}$ for $72 \mathrm{~h} . M$. bovis $B C G$ and $M$. tuberculosis were grown at $37^{\circ} \mathrm{C}$ for 3 weeks on Mycobacteria L-J Culture Medium (Encode Medical Engineering Co. Ltd, Zhuhai, China) before harvesting. E. coli and B. subtilis were cultured in Luria-Bertani media at $37^{\circ} \mathrm{C}$ for $24 \mathrm{~h}$. Heatkilled (dead) M. smegmatis was made by heating at $100^{\circ} \mathrm{C}$ for $10 \mathrm{~min}$. Then, live/heat-killed M. smegmatis were centrifuged and suspended to $1 \times 10^{8}$ colony-forming units (CFUs) $/ \mathrm{ml}$ with RPMI 1640 for infection experiments.

\section{Conditioned Medium Treatment}

Mycobacteria smegmatis were cultured in RPMI 1640 complete medium supplemented with $10 \%$ HS and 5\% FBS, or in DMEM medium supplemented with $10 \%$ FBS. The RPMI 1640 or DMEM without serum was as a CM control. After M. smegmatis were grown at $37^{\circ} \mathrm{C}$ for $48 \mathrm{~h}$ to a final concentration of $1 \times$ $10^{9} \mathrm{CFU} / \mathrm{ml}$, the bacteria culture supernatant was harvested and filtered through a $0.2 \mu \mathrm{m}$ filter membrane as conditioned medium (CM) and used for the incubation of PC12 or C17.2 cells.

\section{Infection Experiments}

Live/heat-killed M. smegmatis were suspended to $1 \times 10^{8}$ CFUs/ml with RPMI 1640 medium. Then, cells were infected with bacteria at a multiplicity of infection (MOI) of 10:1. PC12 or C17.2 cells were co-cultured with M. smegmatis for $48 \mathrm{~h}$ at $37^{\circ} \mathrm{C}$ with $5 \% \mathrm{CO}_{2}$.

\section{Differentiation Induction Experiment}

PC12 or C17.2 cells were seeded in a 6-well plate at the density of $1 \times 10^{6}$ cells/well and incubated for $48 \mathrm{~h}$ until reaching $80 \%$ confluence. Then, cells were treated with $10 \% \mathrm{CM}(\mathrm{v} / \mathrm{v})$ from M. smegmatis, or $0.5 \mathrm{ng} / \mathrm{ml}$ interferon gamma (IFN- $\gamma$ ) (ab645, abcam, USA), or anti-INF $\gamma$ (1:1,000 dilution, AF-585NA, R\&D system, USA) for $48 \mathrm{~h}$. For positive control, PC12 cells were treated with $50 \mathrm{ng} / \mathrm{ml}$ nerve growth factor (NGF Sigma, USA), and C17.2 cells were treated with $50 \mathrm{ng} / \mathrm{ml} \mathrm{NGF}$ and $50 \mathrm{ng} / \mathrm{ml}$ brain-derived neurotrophic factor (BDNF, Sigma, USA. For screening experiment of signal pathway, cells were treated with CM and different inhibitors (AG490, SP600125, U0126, SB239063, PD98059, H89, Y27632, IWP2, DAPT, BAY 11-7082, LY294002, APExBIO Technology, Houston, USA) for $48 \mathrm{~h}$ respectively. The morphology of the cells was observed by microscope (Nikon TE 300).

\section{Cell Viability Assay}

Cell viability was measured using an MTT cell kit (Beyotime Biotechnology, Shanghai, China) following the manufacturer's instructions. Briefly, cells were seeded into 96-well plates at the density of $5 \times 10^{4}$ cells/well and were cultured for $12 \mathrm{~h}$. Then, cells were treated by $\mathrm{CM}$ for the indicated times. The absorbance was measured at $570 \mathrm{~nm}$ by an iMark Microplate Absorbance Reader.

\section{Neurite Outgrowth Measurement}

PC12 or C17.2 cells were treated with CM for $48 \mathrm{~h}$ to analyze neurite outgrowth. Cell morphological changes were observed under a phase contrast microscope at a magnification of $200 \times$. Neurite outgrowth was defined as a process with a length $>2$-fold 
of the cell body. The percentage of cells with neurite outgrowth was quantified for 300 cells/well in randomly chosen fields ( $n$ $=3$ /group). Then, the stimuli were removed, and the cells were cultured in complete cell medium for $12 \mathrm{~h}$, afterwards the percentage of remaining neurite outgrowth cells was calculated again ( $n=3$ /group).

\section{Western Blotting}

Total protein was extracted using a lysis buffer and protease inhibitor (Beyotime Biotechnology, China). Equivalent protein amounts were denatured in an SDS sample buffer, and then were separated by $10 \%$ SDS-PAGE and transferred onto polyvinylidene difluoride membrane. After being blocked with 5\% non-fat dry milk in PBS containing $0.05 \%$ Tween20 , the blotted membranes were incubated with anti-GAP43 antibody, anti-TUBB3 antibody, anti-synaptophysin antibody (1:1,000 respectively, BS3655, BS1345, AP0013, Bioworld, China), anti-Nestin antibody, anti-MAP-2 antibody, anti-P62 antibody (1:1,000 respectively, Abcam, USA), anti-LC3 antibody (1:1,000 respectively, AF5384, AF5402, Affinity, USA) and anti-Phospho-Akt antibody, anti-Akt antibody, anti-PhosphomTOR antibody, anti-mTOR antibody (1:1,000 respectively, 9,271, 9,272, 2,971, 2,972, Cell Signaling Technology, USA) and secondary antibody (1:5,000, Boster, China). $\beta$-tubulin protein levels were also determined by using the specific antibody (1:1,000, BS1842, Bioworld, China) as a loading control.

\section{Immunofluorescence Staining}

Cells were seeded onto glass coverslips and cultivated for $24 \mathrm{~h}$ until reaching $60 \%$ confluence. The medium was replaced with $1 \mathrm{ml}$ of fresh complete medium and 10\% CM (v/v). The cells were then cultivated for $48 \mathrm{~h}$. The cells were washed with PBS three times and then were fixed with $4 \%$ paraformaldehyde. Then, the cells were incubated with primary antibodies (GAP43, synaptophysin, 1:100 respectively) at $4^{\circ} \mathrm{C}$ overnight. Subsequently, the cells were incubated with the FITCconjugated secondary antibody (1:1,000, Boster, China) for $1 \mathrm{~h}$ at room temperature. The nuclei of cells were visualized using 4,6-diamidino-2-phenylindole (DAPI) staining. The cells were imaged at $40 \times$ magnification using the Zeiss LSM710 confocal microscope (Zeiss, Oberkochen, Germany).

\section{Cytokine Analysis}

The quantification of cytokines in the $\mathrm{CM}$ was performed using a $\mathrm{BD}^{\mathrm{TM}}$ Cytometric Bead Array panel kit (BD Biosciences). The analytes included in the 6-plex kit were as follows: IL2 , IL-4, IL-6, IL-10, IFN- $\gamma$, and TNF- $\alpha$. The 6 cytokines were measured by flow cytometry according to the manufacturer's instructions.

\section{Statistical Analysis}

The data was expressed as mean \pm standard deviation (SD) for the indicated experiments. Paired $t$-tests were used to analyze data unless otherwise indicated. A $P<0.05$ was considered statistically significant.

\section{RESULTS}

\section{Co-incubation With M. smegmatis Induced Neuron-Like Morphological Changes of PC12 Cells and C17.2 Cells}

To investigate whether $M$. smegmatis influences the morphology of host cells, PC12 and C17.2 cells were co-incubated with $M$. smegmatis at $37^{\circ} \mathrm{C}$ with $5 \% \mathrm{CO}_{2}$ respectively. Pathogenic $M$. tuberculosis and non-pathogenic $M$. bovis BCG were used as the slow-growing Mycobacteria controls. As shown in Figure 1, both PC12 and C17.2 cells exhibited obvious morphological changes when co-incubated with live $M$. smegmatis compared to streptomycin-treated or heat-killed M. smegmatis at MOI of 10:1 (bacillui: cells) for $48 \mathrm{~h}$, whereas no morphological change were observed when co-incubated with live M. tuberculosis or M. bovis BCG (Figure S1). Surprisingly, the infected PC12 and C17.2 cells showed a neuron-like morphology which is similar to NGF-differentiated PC12 or BDNF-differentiated C17.2 cells.

Moreover, MTT assays revealed that infected PC12 and C17.2 cells exhibited a reduced cell viability in a time-dependent manner and in a dose-dependent manner, but more than $80 \%$ cells were still viable at $48 \mathrm{~h}$ post-infection (MOI:10:1) (Figure 1). These results indicated that cells can survive under the infection condition with M. smegmatis, and live M. smegmatis further induced neuron-like morphological changes in both of non-neuronal PC12 cell line and neural progenitor/stem cells C17.2.

\section{Culture Supernatant of $M$. smegmatis Induced Neuron-Like Morphology Changes of PC12 Cells}

Furthermore, M. smegmatis was observed to proliferate well in the complete cell culture medium (including 10\% FBS in RPMI 1640), whereas cannot grow in serum-free cell medium. M. smegmatis was then cultured for $48 \mathrm{~h}$ in the culture medium with $10 \%$ FBS, and the culture supernatant was collected as a conditioned medium (CM). Gram positive Bacillus subtilis and Gram negative Escherichia coli were cultured in the same culture medium and the supernatant was used as non-Mycobacterium bacteria controls. We observed the neurite-like initiations at $12 \mathrm{~h}$, and its enhanced extensions from 24 to $48 \mathrm{~h}$ after treating the PC12 cells with CMs from $M$. smegmatis (Figure S2). Figures 2A-D showed that $\mathrm{CM}$ induced obviously neuron-like morphological changes in PC12 cells at $48 \mathrm{~h}$, whereas heated supernatant and CM controls from $B$. subtilis or E. coli failed to induce visible changes.

Next, CM was concentrated 5-fold and preliminarily purified by ultrafiltration with the cut-off limit of $10 \mathrm{kD}$. The PC12 cells treated with the ultrafiltrated CM exhibited neuron-like changes, whereas outflow from ultrafiltration membrane did not show any gross morphological alterations (Figures 2E,F). MTT analysis showed that PC12 cells were preserved more than $90 \%$ viability when treated with the ultrafiltrated CM for $48 \mathrm{~h}$ (Figure 2G). However, when $\mathrm{CM}$ was concentrated about 5 -fold by freeze 
A

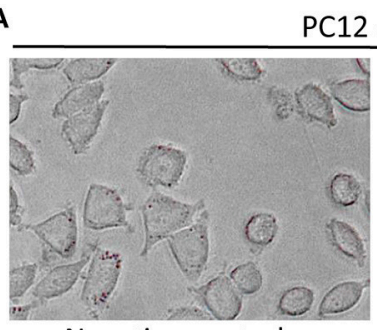

Negative control

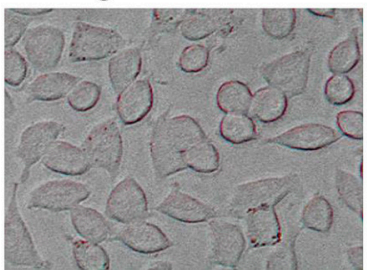

Heated bacteria

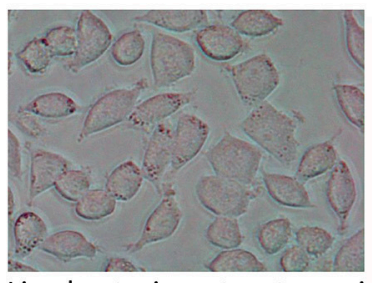

Live bacteria + streptomycin

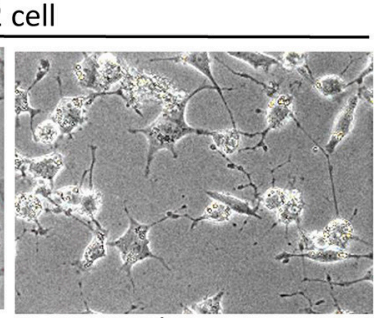

Live bacteria

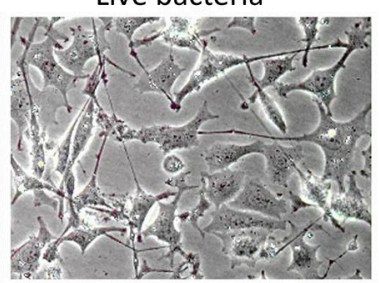

Positive control

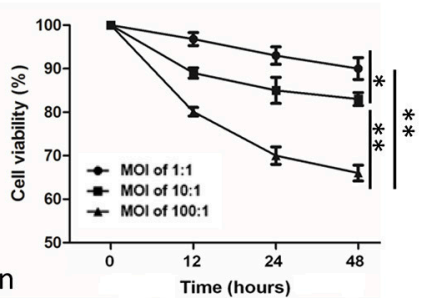

B

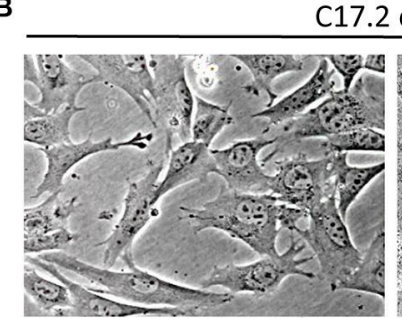

Negative control

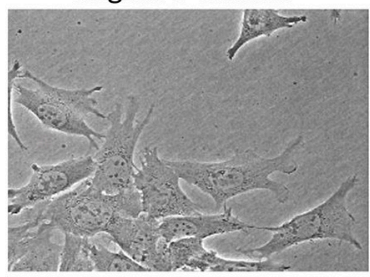

Heated bacteria

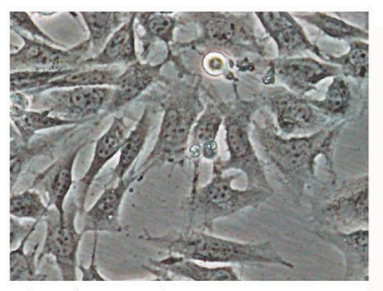

Live bacteria + streptomycin

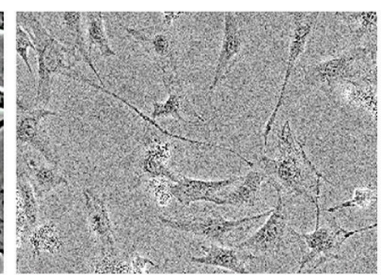

Live bacteria

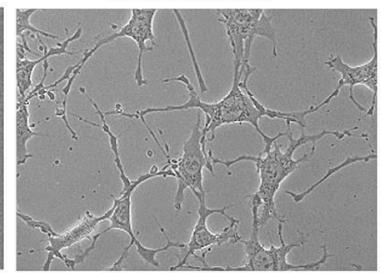

Positive control

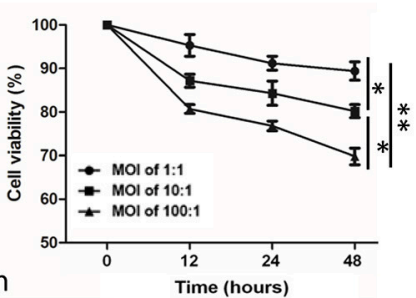

FIGURE 1 | M. smegmatis infection induced morphological changes of PC12 and C17.2 cells. M. smegmatis (MOI 10:1) were added to PC12 (A) or C17.2 (B) cells for $48 \mathrm{~h}$, cell morphologies were observed under different treatments ( $\times 40) .150 \mathrm{ng} / \mathrm{ml} \mathrm{NGF}$ was added in positive control of PC12 cells; $50 \mathrm{ng} / \mathrm{ml} \mathrm{NGF} \mathrm{and} 50 \mathrm{ng} / \mathrm{ml}$ BDNF were added to the positive control of C17.2 cells; Streptomycin (100 $\mu \mathrm{g} / \mathrm{ml})$ and heat-killed $M$. smegmatis (MOI 10:1) were added as dead bacteria controls. Cell viability was measured by an MTT assay with indicated infection dose and time. Quantitative results are presented as means \pm S.D. for 3 replicates. ${ }^{*} P<0.05$, ${ }^{* *} P<0.01$, vs. control group, $n=3$

drying, PC12 cells showed a significantly reduced cell viability (Figure 2H).

These results indicated that the culture supernatant of $M$. smegmatis, but not the bacteria itself, plays a role in the progress of morphological changes, suggesting that M. smegmatis could secrete heat-labile extracellular protein with a molecular weight $>10 \mathrm{kD}$ which induced the neuron-like changes.

\section{Culture Supernatant of $M$. smegmatis Induced Neuronal Differentiation of PC12 Cells and C17.2 Cells}

To further elucidate whether the extracellular protein of $M$. smegmatis induced neuronal differentiation, neurite outgrowth was measured at 2 days post-infection. As shown in Figures 3A,B, a higher frequency of longer neurites as well as a higher average of neurite length were observed in $M$. smegmatis-CM-stimulated PC12 cell, and about a 5-fold increase in neurite length and an 8-fold increase in neurite-bearing cells were determined compared to untreated cells (Figures 3E,F). Similar results were also observed in CM-stimulated C17.2 cells (Figures 3C,D,G,H), suggesting that extracellular proteins of $M$. smegmatis strongly increased neurite outgrowth in PC12 and C17.2 cells.
Additionally, three neural differentiation markers were assessed in CM-stimulated PC12 and C17.2 cells by western blotting and immunofluorescences staining respectively. As shown in Figure 4A, the expression of synaptic markers (GAP43 and SYN) and immature neuronal marker TUBB3 was upregulated in both of CM-stimulated PC12 and C17.2 cells. In addition, the expression of mature neuronal marker MAP2 was up-regulated, whereas neural stem cell marker Nestin was downregulated in CM-stimulated C17.2 cells (Figure 4B). Consistent with results of western blotting in Figure 4, immunofluorescence assays indicated that CM-stimulated PC12 and C17.2 cells exhibited neuron-like morphological changes with strong expression of GAP43 and SYN (Figure 5). These results further identified that neural differentiation of PC12 and C17.2 cells may be induced by the extracellular protein secreted from $M$. smegmatis.

\section{Culture Supernatant of $M$. smegmatis Induced Neuronal Differentiation in Autophagy-Independent Pathways}

Autophagy is involved in the host immunity against bacterial infection. To investigate whether autophagy plays a role in CM-stimulated neural differentiation, the autophagy related 

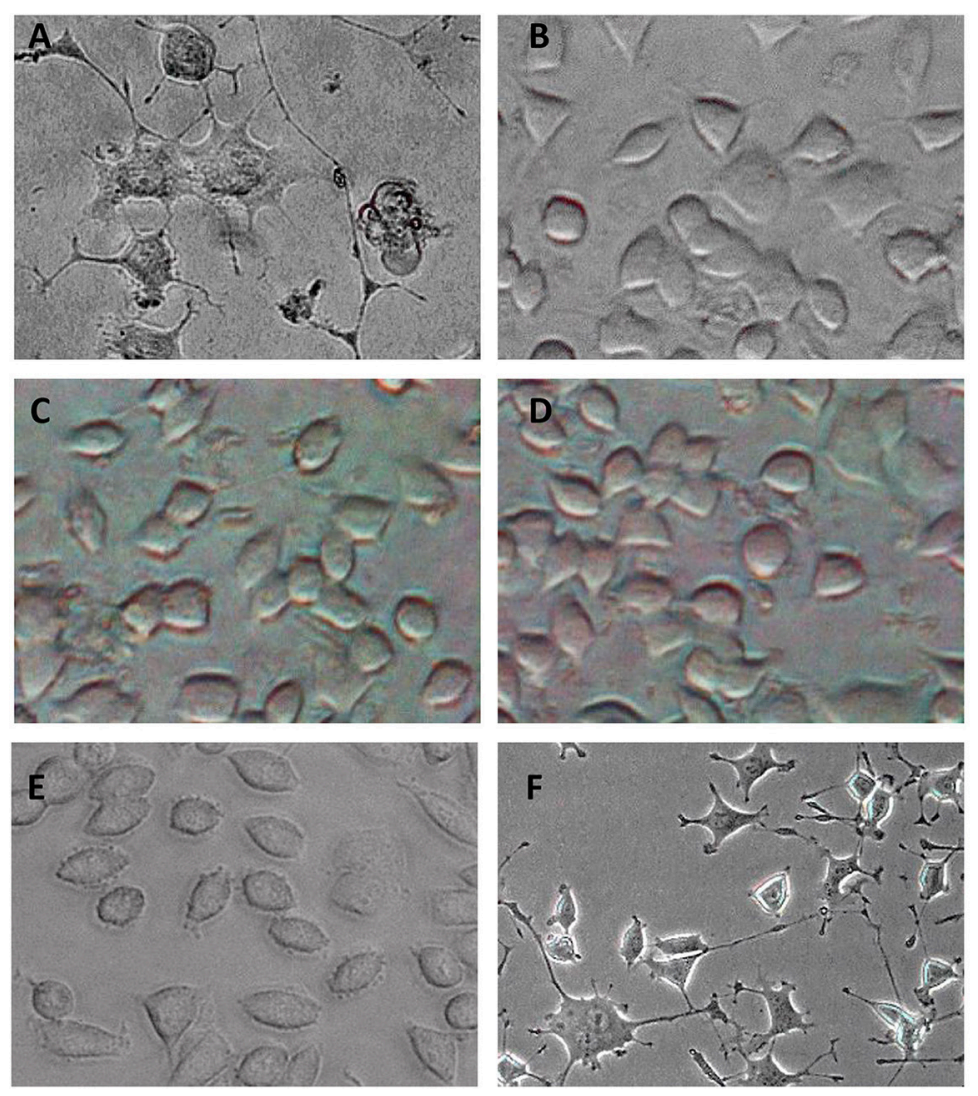
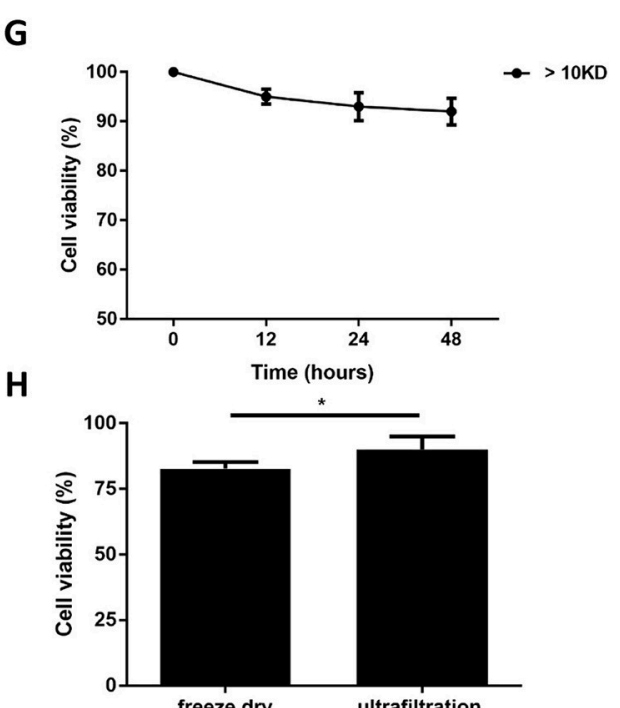

freeze dry

FIGURE 2 | Morphology changes of PC12 cells treated with culture supernatant of $M$. smegmatis. PC12 cells treated with culture supernatant of $M$. Smegmatis (A), B. subtilis (B), E. coli (C), and heat-killed M. smegmatis (D); (E,F) $<10 \mathrm{kD}$ and $>10 \mathrm{kD}$ subgroup of live M. smegmatis culture supernatant for $48 \mathrm{~h}$, and cells were observed under phase-contrast microscope $(\times 40)$. (G) Cell viability of PC12 treated with ultrafiltrated supernatant at indicated time; $(\mathbf{H})$ The relative viability of cells treated by $\mathrm{CM}$ with freeze drying or ultrafiltration for $48 \mathrm{~h}$. Quantitative results were presented as means \pm S.D. for 3 replicates. ${ }^{\star} P<0.05$, vs. control group, $n=3$.
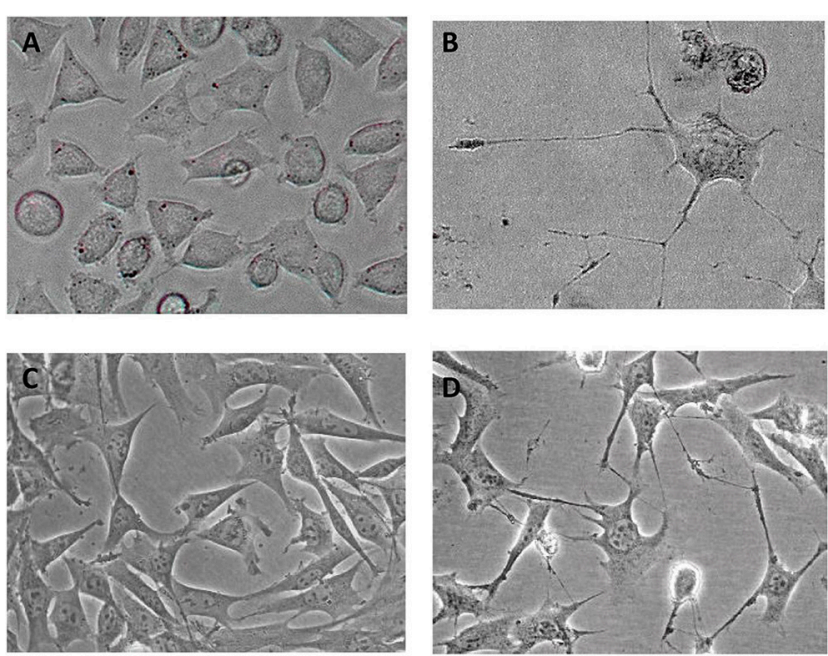

\section{$\mathbf{E}$}

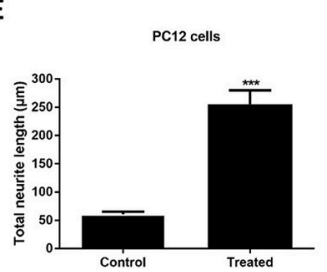

G

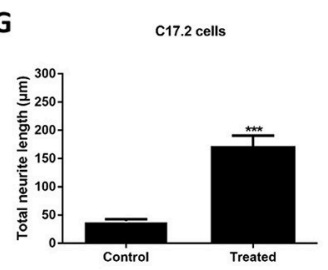

$\mathbf{F}$

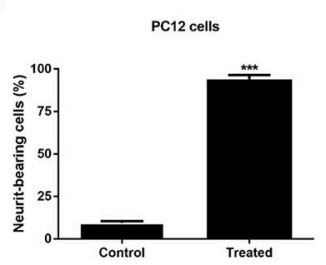

H

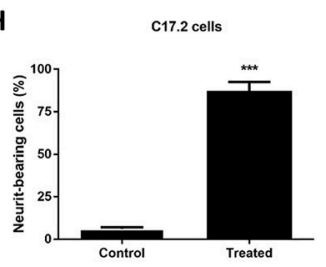

FIGURE 3 | Neurite outgrowth of PC12 cells and C17.2 cells treated with culture supernatant of M. smegmatis. Changes of neurite length in PC12 and C17.2 cells were observed after being stimulated with $M$. smegmatis culture supernatant for $48 \mathrm{~h}$. Cell morphologies of PC12 cells (A,B) and C17.2 cells (C,D) treated with $M$. smegmatis culture supernatant were observed respectively ( $\times 40)$; Neurite length and neurite-bearing cells of PC12 cells (E,F) and C17.2 cells (G,H) treated with $\mathbf{M}$. smegmatis culture supernatant respectively. Quantitative results are presented as means \pm S.D. for 3 replicates. ${ }^{\star \star \star} P<0.001$, vs. control group, $n=3$. 


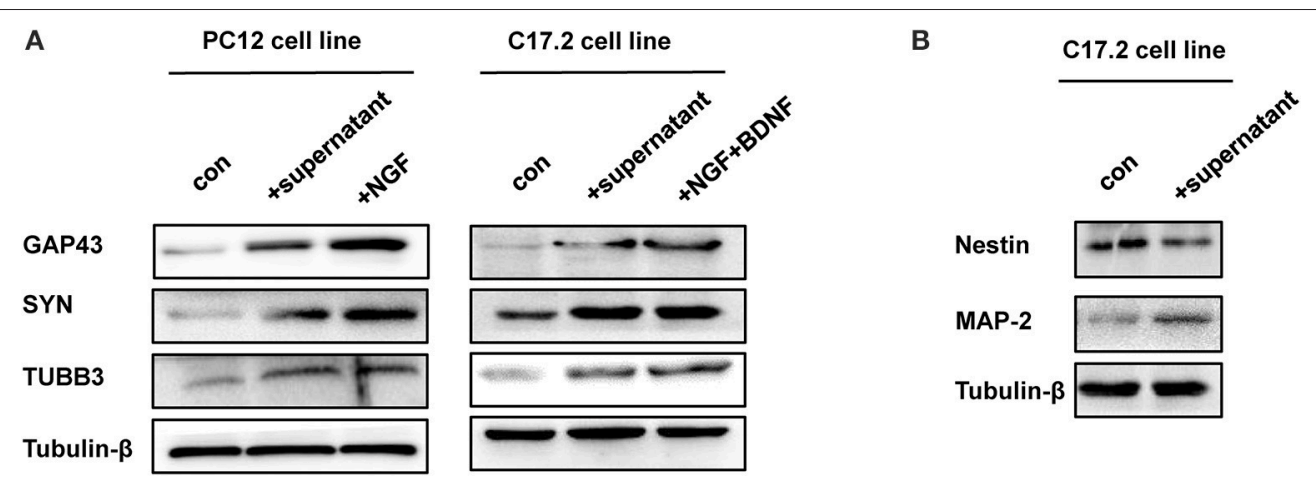

FIGURE 4 | Western blotting analysis of differentiation-related markers of PC12 cells and C17.2 cells treated with culture supernatant of M. smegmatis. (A) PC12 cells and C17.2 cells were treated with/without culture supernatant of M. smegmatis, differentiation related markers GAP43 (Growth Associated Protein 43), SYN (Synaptophysin) and TUBB3 (Tubulin beta-3 chain) were determined by western blot analysis; (B) Neuron related markers Nestin and MAP-2 were determined by western blot analysis in C17.2 cells.

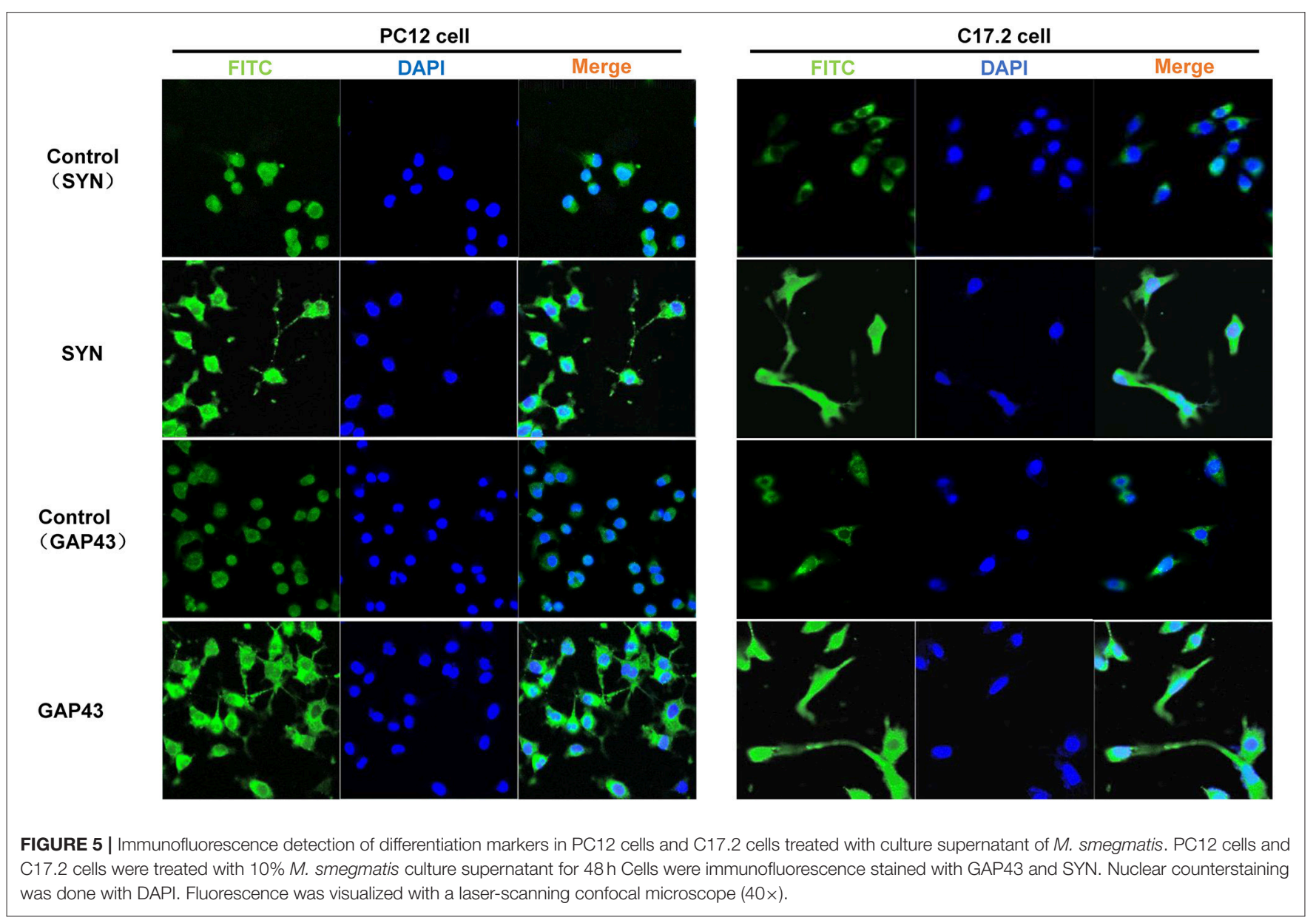

proteins are further detected by Western bolt assay. As shown in Figure 6A, PC12 cells treated with CM obviously induced LC3 I to II conversion, and autophagy inhibitor 3-methyladenine (3-MA) markedly inhibited the CM-induced conversion. Interestingly, $\mathrm{CM}$ and autophagy activator rapamycin exhibited similar ability to induce LC3 I to II conversion. In addition, although no obvious difference was detected in the levels of autophagy receptor p62 between untreated and CMtreated PC12 cells, rapamycin treatment led to a reduced level of p62 in CM-stimulated PC12 cells. These results suggested that culture supernatant of $M$. smegmatis induced autophagy. 

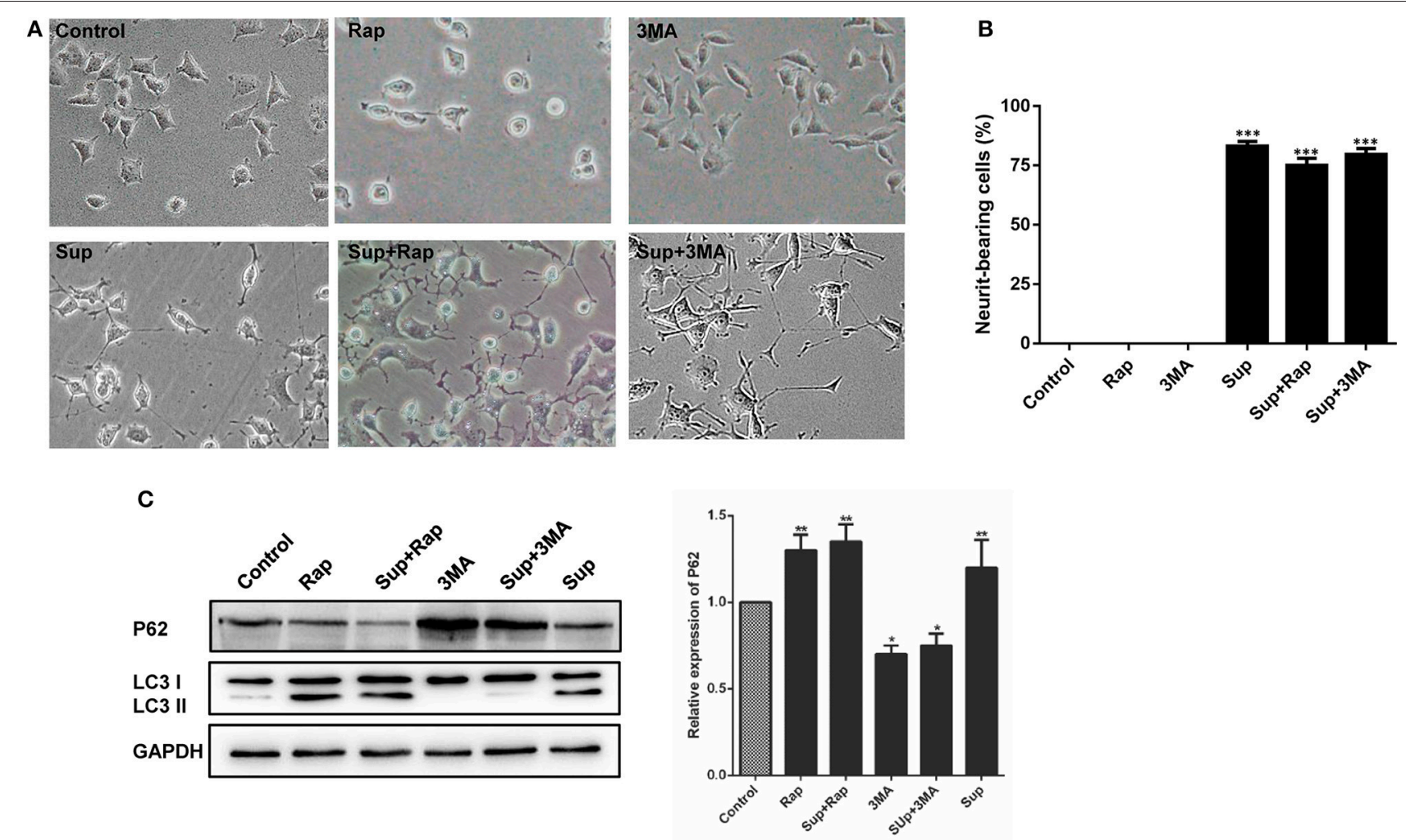

FIGURE 6 | Autophagy did not affect neurite outgrowth in PC12 cells. (A) The expression of the autophagy markers P62 and LC3 were determined by western blot analysis in PC12 cells; (B) PC12 cells morphology changed and (C) neurite-bearing cells counted after treatment with M. smegmatis culture supernatant (Sup), the autophagy inducer rapamycin (1 $\mu \mathrm{M}$, Rap), the autophagy inhibitor 3-MA (10 mM, 3-MA), Sup + Rap, and Sup + 3-MA. (20x) for 48 h. Quantitative results were presented as means \pm S.D. for 3 replicates. ${ }^{\star} P<0.05,{ }^{\star \star} P<0.01,{ }^{\star \star \star} P<0.001$ vs. control group, $n=3$.

Additionally, the neurite-bearing cells were counted in the treated PC12 cells. As shown in Figures $6 \mathbf{B}, \mathbf{C}$, there was no obvious change in neurite outgrowth of PC12 cells neither treated with autophagy activator rapamycin or inhibitor 3-methyladenine (3-MA). The CM-stimulated neural differentiation of PC12 cannot be further affected by autophagy activator or inhibitor. Taken together, these results indicated that culture supernatant of $M$. smegmatis promoted neuronal differentiation in an autophagy-independent pathway.

\section{PI3K-Akt Pathway Is Involved in the Role of M. smegmatis Induced Neuronal Differentiation}

IFN- $\gamma$ is a key immune mediator during mycobacterial infections, and may serve as a common mechanism of differentiation in nervous system (Kim et al., 2007). Therefore, cytokines were measured by flow cytometry using a cytokine bead array. As shown in Figure 7A, IFN- $\gamma$ was detected in the supernatants of PC12 and C17.2 cells treated with CM, while the TNF- $\alpha$, IL- 2, IL- 4 , and IL- 6 concentrations were below the limits of detection until $48 \mathrm{~h}$. In addition, IFN- $\gamma$ levels peaked
$(10.47 \pm 1.34 ; 8.52 \pm 0.65 \mathrm{pg} / \mathrm{ml})$ at $48 \mathrm{~h}$ post-incubation and substantially decreased. Moreover, exogenous IFN- $\gamma$ treatment promoted neuronal differentiation and a neutralizing antibody of IFN- $\gamma$ significantly inhibited either IFN- $\gamma$ or CM-induced differentiation of PC12 and C17.2 cells (Figure 7B).

Then, we screened the signal pathway which might be involved in the differentiation phenomenon through inhibitors of different pathway. Figure S3A showed that PI3K inhibitor LY294002 inhibited the cell differentiation caused by culture supernatant of $M$. smegmatis, and this result was further confirmed by the western blotting (Figure S3B).

IFN- $\gamma$ is an important mediator of inflammation and may activate PI3K-Akt signaling pathways (Nguyen et al., 2001). Next, PC12, and C17.2 cells were further treated with PI3K inhibitor LY294002. As shown in Figure 7C, the CM-induced neurite outgrowth in PC12 and C17.2 was obviously inhibited by LY294002. Additionally, after PC12 cells co-cultured with the CM of M. smegmatis for $48 \mathrm{~h}$, Western blot analysis showed that CM increased phosphorylation of Akt, mTOR and S6K1, while LY294002 inhibited the effects of p-Akt, p-mTOR, and p-S6K1 (Figure 7D).

The data indicated that $M$. smegmatis induced the production of proinflammatory cytokines IFN- $\gamma$, and IFN- $\gamma$ might be 

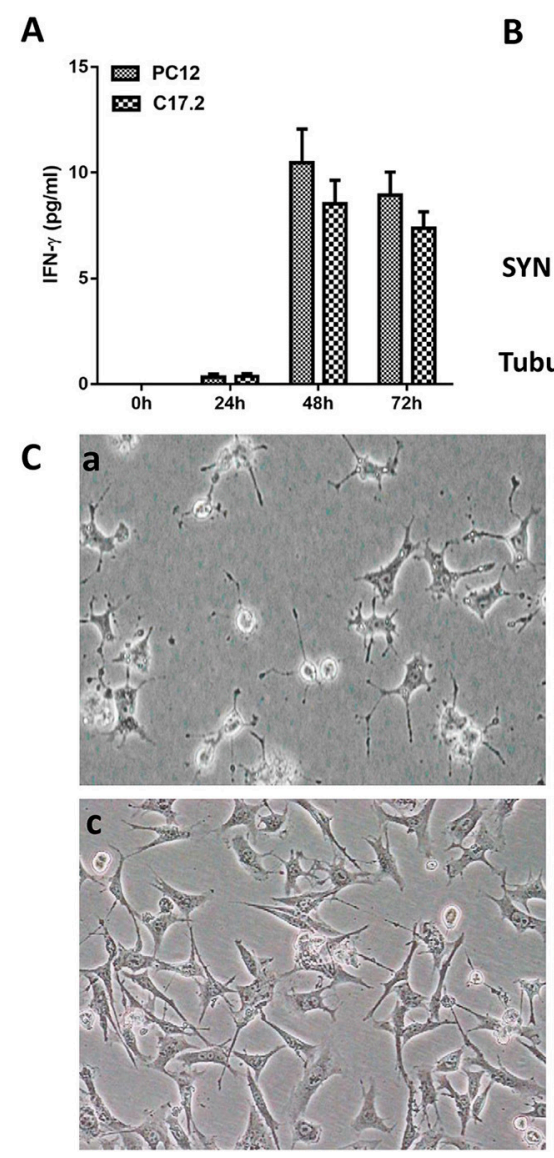

B PC12 cell line

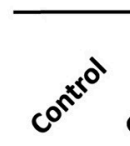

SYN

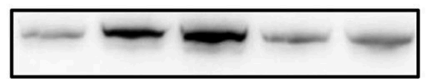

Tubulin $\beta$

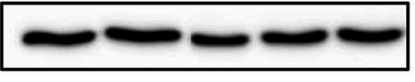

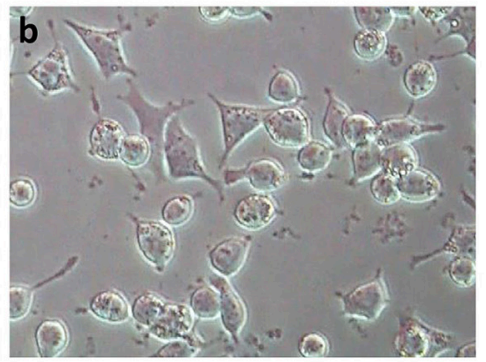

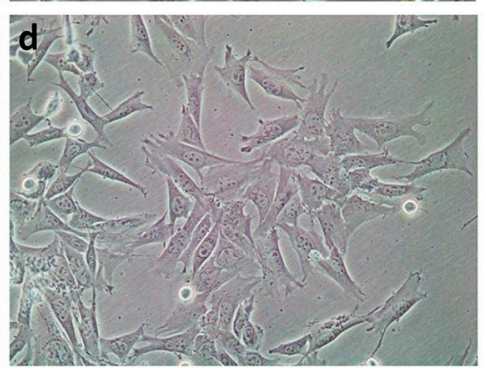

D

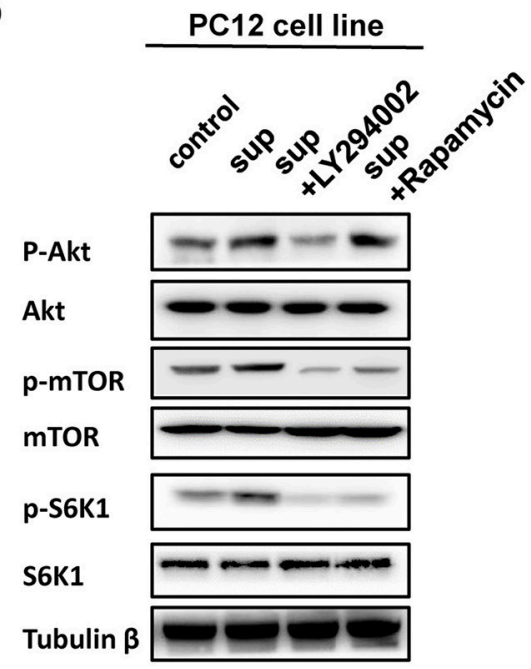

C17.2 cell line
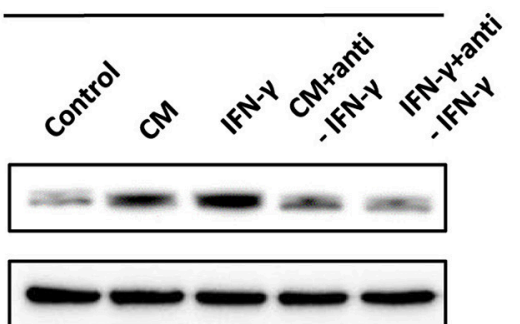

FIGURE 7 | IFN- $\gamma$ involved in the role of $M$. smegmatis induced neuronal differentiation of PC12 cells and C17.2 cells. (A) IFN- $\gamma$ in the supernatant of $M$. smegmatis was measured using a cytokine bead array by flow cytometry; (B) Western blotting analysis of exogenous IFN- $\gamma$ treatment in PC12 and C17.2 cells; (C) PC12 cells were treated with culture supernatant of $M$. smegmatis (a) and supernatant plus LY294002 (b) for 48 h; C17.2 cells were treated with culture supernatant of $M$. smegmatis (c) and supernatant plus LY294002 (d) for 48 h (20×); (D) PC12 cells were exposed to RMPI 1640, supernatant of M. smegmatis, LY29402 for $24 \mathrm{~h}$ respectively. Levels of p-AKT/AKT, p-mTOR/mTOR and p-S6K1/S6K1 were measured by western blot assay.

involved in neuronal differentiation through the activation of the PI3K-Akt signaling pathway.

\section{DISCUSSION}

In this study, we demonstrated an interesting phenomenon that M. smegmatis infection could induce neuron-like morphological changes of PC12 and C17.2 cells. Some microbial and its metabolite can induce neurite outgrowth (Nakashima et al., 2002). Various short-chain fatty acids such as butyrate, induce morphological and biochemical differentiation of glioma or neuroblastoma toward a neuronal phenotype (SuzukiMizushima et al., 2002). Similar results were found in adenosine analog, retinoic acid (Tonge and Andrews, 2010). NO is reported to involve in NGF-induced differentiation of PC12 cells (Oh et al., 2010). In addition, some proteins such as cholera toxin from Vibrio cholerae, p15 protein from Aspergillus oryzae, can induce neurite outgrowth and differentiation in PC12 cells (Glineur and Locht, 1994); Skp, a 17-kDa Escherichia coli molecular chaperone protein, was reported to convert pluripotent P19 cells into functionally active neurons (Halder et al., 2015). In our study, the culture supernatant from the complete medium of fast-growing M. smegmatis but not slow-growing $M$. tuberculosis or $M$. bovis induced neuronal differentiation. Furthermore, the culture supernatant concentrated by ultrafiltration $(10 \mathrm{kD})$ exhibited good activity and was inactivated when heated at $100^{\circ} \mathrm{C}$. Although we couldn't separate and purify the active substances, these results suggested that the effective substances were heatsensitive extracellular proteins, which will be secreted from fastgrowing Mycobacterium with a sufficient concentration to induce neuronal differentiation during the infection in cultured cells

An in-depth study of neural dendrite formation is of great significance both in theories and applications. Interestingly, $M$. smegmatis can cross the modal blood-brain barrier, although the median invasion fraction for M. smegmatis was significantly lower than that for M. tuberculosis (Jain et al., 2006). In general, CNS infection can disrupt the survival, proliferation, and maturation of neural stem precursor cells (NSPC), which ultimately impair neurogenesis. However, the adult mammalian brain creates new neurons from pools of stem cells. Recent studies have shown that neurogenesis increases in response to brain injuries, neurodegenerative conditions and infection. 
For example, although neuronal damage in the hippocampal formation is a common feature of $S$. pneumoniae meningitis, an increased neurogenesis was found after experimental meningitis (Lian et al., 2016). Zika Virus (ZIKV)-infected cranial neural crest cells (CNCCs) undergo limited apoptosis but secrete cytokines that promote death and drive aberrant differentiation of neural progenitor cultures (Bayless et al., 2016).

The neural and the immune systems share many of the same regulatory factors such as IL-6, IFN- $\gamma$, and TNF- $\alpha$ (Thomson et al., 2014). The PI3K-Akt pathway that is stimulated by cytokines, and by its strong links to cell proliferation, survival and inflammation, is one of the most important signaling pathways involved in the regulation of neural functions (Yin et al., 2017; Zhang et al., 2017). Notably, IFN- $\gamma$ plays an especially important role in the innate host response to microbial infections (Warren et al., 2017). Treatment with IFN- $\gamma$ resulted in the increased killing of intracellular bacteria. IFN- $\gamma$ also exerts both protective and pathological effects on other CNS diseases. IFN- $\gamma$ promotes neuronal differentiation of some cells, such as HUCB-derived progenitors (Arien-Zakay et al., 2009). Some studies have shown that anti-viral immunity protects the NSPC population during a neonatal viral CNS infection by IFN$\gamma$ (Fantetti et al., 2016). Our study further demonstrated that IFN- $\gamma$ displayed the role of neuronal differentiation during $M$. smegmatis stimulation by PI3K-Akt signaling pathways. In line with our study, IFN- $\gamma$ was reported to facilitate NGF-induced neuronal differentiation in PC12 cells (Improta et al., 1988), and to regulate proliferation and neuronal differentiation by STAT1 in normal adult brains (Pereira et al., 2015). Furthermore, a recent study has reported that neonatal BCG vaccination and BCG-serum promote hippocampal neurogenesis and cognitive function in early life, accompanied with elevated IFN- $\gamma$ levels both in the periphery and the brain (Yang et al., 2016).

Moreover, multiple studies have indicated a role of autophagy in neuronal differentiation (Li et al., 2014; Mirzaa et al., 2016; Fidaleo et al., 2017). Autophagy can cause neuronal differentiation (Xu et al., 2015). In our study, the culture supernatant of $M$. smegmatis promoted autophagy signaling. Besides, it is well-known starvation could induce cell autophagy, but starvation cannot induce the differentiation in our study. This results further suggested that neuronal differentiation was induced in an autophagy-independent pathway. Moreover, autophagosomes are formed in response to a number of environmental stimuli, and IFN- $\gamma$ has been shown to induce autophagy. Moreover, autophagy can regulate the production and secretion of cytokines, including IFN- $\gamma$ (Harris et al., 2017). Induction of autophagy has been proposed as a reasonable strategy to help neurons clear abnormal protein aggregates and survive (Heras-Sandoval et al., 2014).

M. smegmatis is a rapidly growing environmental species that is present in skin. Although little is known about skin microbiota, this may be the next frontier in elucidating molecular mechanisms of pathogenesis and symbiosis. The skin participates in the stress response by a local hypothalamic-pituitary-adrenal axis (HPA), peripheral nerve endings and skin cells. Proinflammatory cytokines and neurogenic inflammatory pathways involve in the brain-skin connection (Chen and Lyga, 2014). $M$. smegmatis and M. tuberculosis share many features and identical genomic sequences are a potential candidate for developing new tuberculosis vaccines (Nguyen Thi et al., 2010). It is reported that fatal disseminated $M$. smegmatis infection in a child with inherited IFN- $\gamma$ receptor deficiency (Pierre-Audigier et al., 1997). In addition, administration of live M. smegmatis was administered to mice to produce Th1-type cellular responses (IFN- $\gamma$ and IL-2) and anti-tumor effects (Young et al., 2004). These suggested the non-pathogen Mycobacterium could be used to promote neuronal differentiation in neurodegenerative disorders.

In summary, M. smegmatis infection can induce neuronal differentiation in an autophagy-independent manner in vitro. Although the details of molecular mechanisms of the effects remain to be elucidated, our findings suggest that live nonpathogenic Mycobacterium infections might not only play the role against intracellular infections, but also prompt neuronal differentiation. In particular, our findings may shed new light on the roles of non-pathogen Mycobacterium such as M. smegmatis in CNS, and may open a novel approach to treating brain tumors or neurodegenerative diseases such as Alzheimer's and Parkinson's diseases. Further studies are going to be conducted in vivo to investigate the effects of live M. smegmatis infections in mice brain and $\mathrm{AD}$ transgenic mice.

\section{AUTHOR CONTRIBUTIONS}

GZ, RP, and XF contributed to the conception and design of the study. GZ and XF contributed to data acquisition, data analysis and manuscript writing. JL, $\mathrm{ZH}, \mathrm{YW}$, and FQ participated in data analysis and manuscript writing. All authors read and approved the final manuscript.

\section{ACKNOWLEDGMENTS}

This work was supported by the National Natural Science Foundation of China (No. 81673005).

\section{SUPPLEMENTARY MATERIAL}

The Supplementary Material for this article can be found online at: https://www.frontiersin.org/articles/10.3389/fcimb. 2018.00201/full\#supplementary-material 


\section{REFERENCES}

Arien-Zakay, H., Lecht, S., Bercu, M. M., Amariglio, N., Rechavi, G., Galski, H., et al. (2009). Interferon-[gamma]-induced neuronal differentiation of human umbilical cord blood-derived progenitors. Leukemia 23, 1790-1800. doi: 10.1038/leu.2009.106

Bayless, N. L., Greenberg, R. S., Swigut, T., Wysocka, J., and Blish, C. A. (2016). Zika virus infection induces cranial neural crest cells to produce cytokines at levels detrimental for neurogenesis. Cell Host Microbe 20, 423-428. doi: 10.1016/j.chom.2016.09.006

Branton, W. G., Ellestad, K. K., Maingat, F., Wheatley, B. M., Rud, E., Warren, R. L., et al. (2013). Brain microbial populations in HIV/AIDS: alpha-proteobacteria predominate independent of host immune status. PLoS ONE 8:e54673. doi: 10.1371/journal.pone.0054673

Chen, Y., and Lyga, J. (2014). Brain-skin connection: stress, inflammation and skin aging. Inflamm. Allergy Drug Targets 13, 177-190. doi: 10.2174/1871528113666140522104422

Fantetti, K. N., Gray, E. L., Ganesan, P., Kulkarni, A., and O'Donnell, L. A. (2016). Interferon gamma protects neonatal neural stem/progenitor cells during measles virus infection of the brain. J. Neuroinflammation 13:107. doi: $10.1186 /$ s12974-016-0571-1

Fidaleo, M., Cavallucci, V., and Pani, G. (2017). Nutrients, neurogenesis and brain ageing: from disease mechanisms to therapeutic opportunities. Biochem. Pharmacol. 141, 63-76. doi: 10.1016/j.bcp.2017.05.016

Glineur, C., and Locht, C. (1994). Importance of ADP-ribosylation in the morphological changes of PC12 cells induced by cholera toxin. Infect. Immun. 62, 4176-4185.

Halder, D., Chang, G. E., De, D., Cheong, E., Kim, K. K., and Shin, I. (2015). Combining suppression of stemness with lineage-specific induction leads to conversion of pluripotent cells into functional neurons. Chem. Biol. 22, 1512-1520. doi: 10.1016/j.chembiol.2015.10.008

Harris, J., Lang, T., Thomas, J. P. W., Sukkar, M. B., Nabar, N. R., and Kehrl, J. H. (2017). Autophagy and inflammasomes. Mol. Immunol. 86, 10-15. doi: 10.1016/j.molimm.2017.02.013

Heras-Sandoval, D., Pérez-Rojas, J. M., Hernández-Damián, J., and PedrazaChaverri, J. (2014). The role of PI3K/AKT/mTOR pathway in the modulation of autophagy and the clearance of protein aggregates in neurodegeneration. Cell Signal. 26, 2694-2701. doi: 10.1016/j.cellsig.2014.08.019

Improta, T., Salvatore, A. M., Di Luzio, A., Romeo, G., Coccia, E. M., and Calissano, P. (1988). IFN-gamma facilitates NGF-induced neuronal differentiation in PC12 cells. Exp. Cell Res. 179, 1-9. doi: $10.1016 / 0014-4827(88) 90342-4$

Jain, S. K., Paul-Satyaseela, M., Lamichhane, G., Kim, K. S., and Bishai, W. R. (2006). Mycobacterium tuberculosis invasion and traversal across an in vitro human blood-brain barrier as a pathogenic mechanism for central nervous system tuberculosis. J. Infect. Dis. 193, 1287-1295. doi: 10.1086/502631

Kim, S. J., Son, T. G., Kim, K., Park, H. R., Mattson, M. P., and Lee, J. (2007). Interferon- $\gamma$ promotes differentiation of neural progenitor cells via the JNK pathway. Neurochem. Res. 32, 1399-1406. doi: 10.1007/s11064-007-9323-Z

Li, Y., Wang, C., Zhang, G., Wang, X., Duan, R., Gao, H., et al. (2014). Role of autophagy and mTOR signaling in neural differentiation of bone marrow mesenchymal stem cells. Cell Biol. Int. 38, 1337-1343. doi: 10.1002/cbin.10320

Lian, D., He, D., Wu, J., Liu, Y., Zhu, M., Sun, J., et al. (2016). Exogenous BDNF increases neurogenesis in the hippocampus in experimental Streptococcus pneumoniae meningitis. J. Neuroimmunol. 294, 46-55. doi: 10.1016/j.jneuroim.2016.03.014

Marion, E., Song, O. R., Christophe, T., Babonneau, J., Fenistein, D., Eyer, J., et al. (2014). Mycobacterial toxin induces analgesia in buruli ulcer by targeting the angiotensin pathways. Cell 157, 1565-1576. doi: 10.1016/j.cell.2014.04.040

Martino, A., Sacchi, A., Volpe, E., Agrati, C., De Santis, R., Pucillo, L. P., et al. (2005). Non-pathogenic Mycobacterium smegmatis induces the differentiation of human monocytes directly into fully mature dendritic cells. J. Clin. Immunol. 25, 365-375. doi: 10.1007/s10875-005-4188-x

Masaki, T., Qu, J., Cholewa-Waclaw, J., Burr, K., Raaum, R., and Rambukkana, A. (2013). Reprogramming adult Schwann cells to stem cell-like cells by leprosy bacilli promotes dissemination of infection. Cell 152, 51-67. doi: 10.1016/j.cell.2012.12.014
Mirzaa, G. M., Campbell, C. D., Solovieff, N., Goold, C., Jansen, L. A., Menon, S., et al. (2016). Association of MTOR mutations with developmental brain disorders, including megalencephaly, focal cortical dysplasia, and pigmentary mosaicism. JAMA Neurol. 73, 836-845. doi: 10.1001/jamaneurol.2016.0363

Nakashima, S., Arioka, M., and Kitamoto, K. (2002). Functional expression in Aspergillus oryzae of $\mathrm{p} 15$, a protein with potent neurite-inducing activity in PC12 cells. Biosci. Biotechnol. Biochem. 66, 674-678. doi: 10.1271/bbb.66.674

Nguyen, H., Ramana, C. V., Bayes, J., and Stark, G. R. (2001). Roles of phosphatidylinositol 3-kinase in interferon- $\gamma$-dependent phosphorylation of STAT1 on serine 727 and activation of gene expression. J. Biol. Chem. 276, 33361-33368. doi: 10.1074/jbc.M105070200

Nguyen Thi, L. T., Maura, R. B., Férnandez, S., Reyes, G., Perez, J. L., Reyes, F., et al. (2010). Evaluation of the potential of Mycobacterium smegmatis as vaccine Candidate against tuberculosis by in silico and in vivo studies. VacciMonitor 19, $20-26$.

Obata, Y., and Pachnis, V. (2016). The effect of microbiota and the immune system on the development and organization of the enteric nervous system. Gastroenterology 151, 836-844. doi: 10.1053/j.gastro.2016.07.044

Ochoa-Repáraz, J., and Kasper, L. H. (2014). Gut microbiome and the risk factors in central nervous system autoimmunity. FEBS Lett. 588, 4214-4222. doi: 10.1016/j.febslet.2014.09.024

Oh, S. J., Heo, J. I., Kho, Y. J., Kim, J. H., Kang, H. J., Park, S. H., et al. (2010). Nitric oxide is an essential mediator for neuronal differentiation of rat primary cortical neuron cells. Exp. Neurobiol. 19, 83-89. doi: 10.5607/en.2010.19.2.83

Oleskin, A. V., Shenderov, B. A., and Rogovsky, V. S. (2017). Role of neurochemicals in the interaction between the microbiota and the immune and the nervous system of the host organism. Probiotics Antimicrob. Proteins 9, 215-234. doi: 10.1007/s12602-017-9262-1

Pereira, L., Medina, R., Baena, M., Planas, A. M., and Pozas, E. (2015). IFN gamma regulates proliferation and neuronal differentiation by STAT1 in adult SVZ niche. Front. Cell Neurosci. 9:270. doi: 10.3389/fncel.2015.00270

Pierre-Audigier, C., Jouanguy, E., Lamhamedi, S., Altare, F., Rauzier, J., Vincent, V., et al. (1997). Fatal disseminated Mycobacterium smegmatis infection in a child with inherited interferon gamma receptor deficiency. Clin. Infect. Dis. 24, 982-984. doi: 10.1093/clinids/24.5.982

Rambukkana, A. (2010). Usage of signaling in neurodegeneration and regeneration of peripheral nerves by leprosy bacteria. Prog. Neurobiol. 91, 102-107. doi: 10.1016/j.pneurobio.2009.12.002

Reber, S. O., Siebler, P. H., Donner, N. C., Morton, J. T., Smith, D. G., Kopelman, J. M., et al. (2016). Immunization with a heat-killed preparation of the environmental bacterium Mycobacterium vaccae promotes stress resilience in mice. Proc. Natl. Acad. Sci. U.S.A. 113, E3130-E3139. doi: $10.1073 /$ pnas. 1600324113

Sharon, G., Garg, N., Debelius, J., Knight, R., Dorrestein, P. C., and Mazmanian, S. K. (2014). Specialized metabolites from the microbiome in health and disease. Cell Metab. 20, 719-730. doi: 10.1016/j.cmet.2014.10.016

Spanos, J. P., Hsu, N. J., and Jacobs, M. (2015). Microglia are crucial regulators of neuro-immunity during central nervous system tuberculosis. Front. Cell Neurosci. 9:182. doi: 10.3389/fncel.2015.00182

Suzuki-Mizushima, Y., Gohda, E., Okamura, T., Kanasaki, K., and Yamamoto, I. (2002). Enhancement of NGF- and cholera toxin-induced neurite outgrowth by butyrate in PC12 cells. Brain Res. 951, 209-217. doi: 10.1016/S0006-8993(02)03163-3

Takahashi, C., Shirakawa, J., Tsuchidate, T., Okai, N., Hatada, K., Nakayama, H., et al. (2012). Robust production of gamma-amino butyric acid using recombinant Corynebacterium glutamicum expressing glutamate decarboxylase from Escherichia coli. Enzyme Microb. Technol. 51, 171-176. doi: 10.1016/j.enzmictec.2012.05.010

Thomson, C. A., McColl, A., Cavanagh, J., and Graham, G. J. (2014). Peripheral inflammation is associated with remote global gene expression changes in the brain. J. Neuroinflammation 11:73. doi: 10.1186/1742-2094-11-73

Tonge, P. D., and Andrews, P. W. (2010). Retinoic acid directs neuronal differentiation of human pluripotent stem cell lines in a non-cell-autonomous manner. Differentiation 80, 20-30. doi: 10.1016/j.diff.2010.04.001

Vuong, H. E., Yano, J. M., Fung, T. C., and Hsiao, E. Y. (2017). The microbiome and host behavior. Annu. Rev. Neurosci. 40, 21-49. doi: 10.1146/annurev-neuro-072116-031347 
Warren, E., Teskey, G., and Venketaraman, V. (2017). Effector mechanisms of neutrophils within the innate immune system in response to Mycobacterium tuberculosis infection. J. Clin. Med. 6:E15 doi: 10.3390/jcm6020015

Xu, P., Li, Z., Wang, H., Zhang, X., and Yang, Z. (2015). Triptolide inhibited cytotoxicity of differentiated PC12 cells induced by Amyloid$\operatorname{Beta}(2)(5)(-)(3)(5)$ via the autophagy pathway. PLoS ONE 10:e0142719. doi: 10.1371/journal.pone.0142719

Yang, J., Qi, F., Gu, H., Zou, J., Yang, Y., Yuan, Q., et al. (2016). Neonatal BCG vaccination of mice improves neurogenesis and behavior in early life. Brain Res. Bull. 120, 25-33. doi: 10.1016/j.brainresbull.2015.10.012

Yin, K., Wang, L., Zhang, X., He, Z., Xia, Y., Xu, J., et al. (2017). Netrin1 promotes gastric cancer cell proliferation and invasion via the receptor neogenin through PI3K/AKT signaling pathway. Oncotarget 8, 51177-51189. doi: 10.18632/oncotarget.17750

Young, S. L., Murphy, M., Zhu, X. W., Harnden, P., O’Donnell, M. A., James, K., et al. (2004). Cytokine-modified Mycobacterium smegmatis as a novel anticancer immunotherapy. Int. J. Cancer 112, 653-660. doi: 10.1002/ijc.20442
Zhang, X., He, X., Li, Q., Kong, X., Ou, Z., Zhang, L., et al. (2017). PI3K/AKT/mTOR signaling mediates valproic acid-induced neuronal differentiation of neural stem cells through epigenetic modifications. Stem Cell Rep. 8, 1256-1269. doi: 10.1016/j.stemcr.2017.0 4.006

Conflict of Interest Statement: The authors declare that the research was conducted in the absence of any commercial or financial relationships that could be construed as a potential conflict of interest.

Copyright (c) 2018 Feng, Lu, He, Wang, Qi, Pi and Zhang. This is an open-access article distributed under the terms of the Creative Commons Attribution License (CC $B Y)$. The use, distribution or reproduction in other forums is permitted, provided the original author(s) and the copyright owner are credited and that the original publication in this journal is cited, in accordance with accepted academic practice. No use, distribution or reproduction is permitted which does not comply with these terms. 